Vol. 4, Special Issue 2, January 2017

\title{
Data Transfer using Visible Light Communication
}

\author{
Mr. Yogesh Chavan ${ }^{1}$, Mr. Ramchandra Gurav \\ Student, Bachelor of Engineering, Electronics \& Telecommunication Engineering Department, Dr. Daulatrao Aher \\ College of Engineering, Karad, Maharashtra, India ${ }^{1}$ \\ Assistant Professor, Electronics \& Telecommunication Engineering Department, Dr. Daulatrao Aher College of \\ Engineering, Karad, Maharashtra, India ${ }^{2}$
}

\begin{abstract}
The visible light communication (VLC) refers to the communication technology which uses the visible light source as a signal transmitter, the air is used as the transmission medium, and the appropriate photodiode as a signal receiving component. Visible light should be considered as the medium for wireless transmission because it has got few advantages over other standard wireless transmissions. LED's can be switched on and off faster, which helps for data transmission. To encode data in the light can be done by varying the rate at which the light flicker ON and OFF to give different strings of $1 \mathrm{~s}$ and $0 \mathrm{~s}$. The intensity of the light is modulated so rapidly that human eye can't detect, so the output appears to be constant. The photo detector at receiver side receives different strings of $1 \mathrm{~s}$ and $0 \mathrm{~s}$ and receiver decodes it in its original form. This data then can be saved to receiver computer. In this way data can be transferred from one computer to another computer.
\end{abstract}

Keywords: Visible Light Communication (VLC), LED, Light Sensor (Photodiode), Data Transmission

\section{INTRODUCTION}

Communication is the essential part in the field of ones and the original information will be recovered. This electronics and communication. It deals with transfer of type of communication has several advantages respect to data from one place to another place. Communication the RF wireless communications, such as free use of the medium has major role in the successful data transfer and visible spectrum, increased security in communication, the to determine the mode of transmission. bandwidth $300 \mathrm{THz}$ and null electromagnetic interference [3]. It has a major advantage that it causes no interference

There are two mode of transmission; wired and wireless transmission. In wired transmission, data is transferred through a physical medium or a link whereas no physical link is used in wireless transmission. Both mediums have it's own characteristics and advantages.

Wireless communication uses the RF source to modulate. But it takes some time. But, if we use a visible light instead of RF wave source, transmission speed can be increased. The visible light communication (VLC) refers to the communication technology which uses the visible light source as a signal transmitter, the air is used as the transmission medium, and the appropriate photodiode as a signal receiving component. to RF-based devices.

This made wireless communication possible in RF hazardous areas such as hospitals and space station. In addition to these two key advantages, safety, simple installation procedures and band licensing-free characteristic also helped to increase VLC's potential to be developed as an alternative, or even a new standard to the wireless communication scheme.

\section{PROPOSED SYSTEM}

The basic principle of working of system is based on conversion of data to be transmitted into TTL form using MAX232 IC. This logic is then applied to LED which will

Visible light should be considered as the medium for wireless transmission because it has got few advantages over other standard wireless transmissions. VLC uses white Light Emitting Diodes (LED), which send data by flashing light at speed. VLC uses white Light Emitting Diodes (LED), which sends data by flashing light at speeds undetectable to the human eye [1]. When signals reach the receiver through the indoor wireless channel, the photodiode will convert the optical signals to electrical

The switching period of LED is fast so it can't be detectable to human eyes. The photodiode at receiving side will capture all the light from LED and fed to MAX232 IC which will convert this data (which is in the form of $0 \mathrm{~s}$ and $1 \mathrm{~s}$ ) into its original format. For applying data to the transceiver circuit USB to serial converter cable can be used. 
Block Diagram:

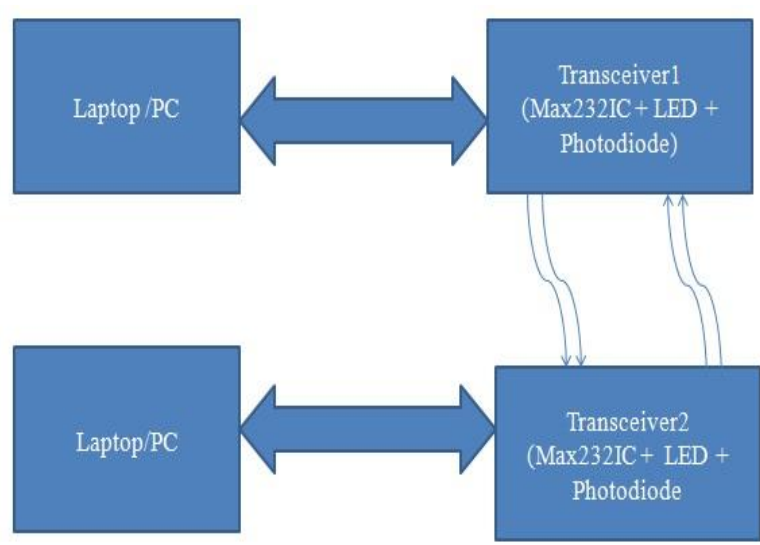

Fig.1. Block Diagram of Proposed system

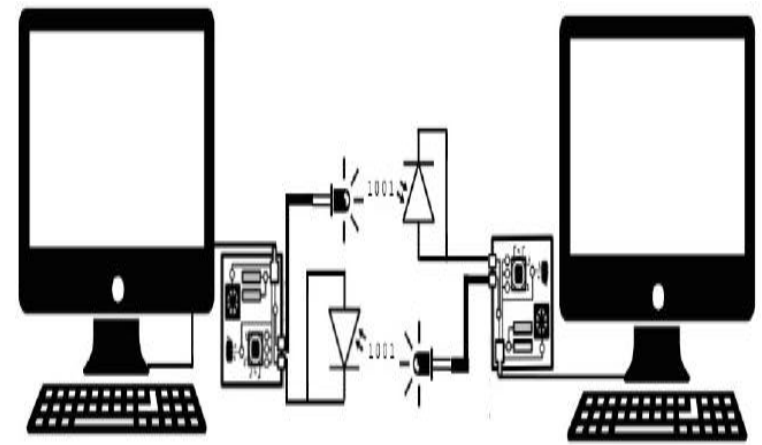

Fig.2. Conceptual diagram for Data transfer between two computers.

\section{WORKING}

For this particular project there are two transceiver circuits. As it is transceiver there are two parts of its working viz. transmitter section and receiver section. Following diagram shows the conceptual diagram of the two transceiver circuits.

\section{A. Transmitter:}

Every kind of light source can theoretically be used as transmitting device for VLC. However, some are better suited than others. For instance, incandescent lights quickly break down when switched on and off frequently. These are thus not recommended as VLC transmitters. More promising alternatives are fluorescent lights and LEDs. VLC transmitters are usually also used for providing illumination of the rooms in which they are used. This makes fluorescent lights and LED particularly popular choice, because they can flicker quickly enough to transmit a meaningful amount of data and are already widely used for illumination purposes. However, with an ever-rising market share of LEDs and further technological improvements such as higher brightness and spectral clarity, LEDs are expected to replace fluorescent lights as illumination sources and VLC transmitters.
Switching time of LED is very high therefore LED is the best choice for the VLC transmitter.

\section{B. Receiver:}

The receiver consists of an optical element to collect and concentrate the radiation onto the receiver photo detector; photodiode convert visible light into an electrical signal biased. The photodiode operates in the photoconductive mode generating a current proportional to the collected light. The resulting voltage is then applied to a low-pass filter to remove any high frequency noise (Here we are using MAX232 IC for this purpose). The resulting voltage signal is then further amplified in the final voltage amplifier stage. Amplifying and filtering stages, which helps reduce the DC noise component of the captured signal as well as low-frequency components. The final voltage signal should correspond to the received light pulses which are then decoded in the final decoder block, thus extracting the digital data. This final block performs the inverse function of the emitter's encoder block, but it can also be implemented with a microprocessor.

\section{HARDWARE REQUIREMENTS}
1) Laptops
2) Power supply of $+5 \mathrm{~V}$.
3) RS232 female connectors.
4) USB cable with RS232 male connector
5) MAX232 IC.
6) LEDs.
7) Photo detectors.
8) Connecting Wires.
9) Buzzers.

\section{A. Power supply:}

For our project we require a $+5 \mathrm{v}$ power supply. This power supply can be designed using voltage regulator IC 7805 .

\section{B. MAX232 IC}

The MAX232 device is a dual driver/receiver that includes a capacitive voltage generator using four capacitors to supply TIA/EIA-232-F voltage levels from a single 5-V supply. Each receiver converts TIA/EIA-232-F inputs to $5 \mathrm{~V}$ TTL/CMOS levels. These receivers have a typical threshold of $1.3 \mathrm{~V}$, a typical hysteresis of $0.5 \mathrm{~V}$, and can accept $\pm 30-\mathrm{V}$ inputs. Each driver converts TTL/CMOS input levels into TIA/EIA-232-F levels.

Features of MAX 232:

- Operates From a Single 5-V Power Supply.

- Two Drivers and Two Receivers.

- $\pm 30-V$ Input Levels.

- Requires low Supply Current 8 mA typical.

C. USB cable with RS232 male connector, RS232 female connector:

RS-232 is a single ended electronic data communication between the DTE (data terminal equipment) and DCE 
(data circuit terminating equipment) in computer serial ports. It supports the bit transmission rate up to 115,200 bps in serial communications. It is the replacement of serial and parallel port communications with more efficiency and ease of use that supports a data rate of $12 \mathrm{Mbps}$ (USB 1.0), 480Mbps (USB 2.0). The new version of USB 3.0 can run up to $5 \mathrm{Gps}$. USB was designed in such a way that it can connect easily to all the computer peripheral devices. It is a hot plug and play with $+5 \mathrm{v}$ at the source.

\section{LED:}

For this particular project LED is used to transmit data converted to TTL form towards the receiving circuit.

\section{E. Photodiode:}

A photodiode converts the incident light into the current. It works on the principle called photo-conduction, whereas LED works on the principle of electro-luminance. The photodiode is a type of photo detector which converts the light to either current or voltage. Photodiode is used to receive data in the form of light transmitted from LED. Photodiode captures all the light and send it towards MAX232 IC which will encode this data into its original form i.e. data will be received at receiver.

\section{F. Buzzer:}

In this project we are using buzzer as a indicator which will indicate transmission of data is in process.

\section{SOFTWARE REQUIREMENTS}

Driver for USB to serial port converter cable. The driver $\mathrm{CD}$ will be provided along with the cable or user have to download driver for particular from its manufacturing site.

\section{APPLICATIONS AND FUTURE SCOPE}

As the data transfer using light is possible without any kind of modulation, this idea can be used in the development of Li-Fi technology by proper design changes in circuits.

This method of data transmission can be applied where optic fiber and radiation prohibited areas such as chemical plants. This method can be used for wireless communication such as communication between space shuttles etc. This analytic study can be used for the future development of visible light communication systems. This can be applied at the chemical plants where the RF waves and OFC cannot be used.

This system you can used into the school, college, lab, hospital, aircraft, air plane, to commanding the robot, mobile to mobile communication, etc. where the RF is ban on some areas and RF is strictly unused on that range like petrol pump which is RF is cause the explosion on this areas.

\section{CONCLUSION}

The LED-to-LED communication provides a unique opportunity to provide communication capabilities that is not noticed. From the simulation we can see that it is possible to transmit higher quality of data using visible light as a medium.

\section{REFERENCES}

[1] Amrutha.S, Ansu Mathew, Rajasree.R, Swathy Sugathan; Aravind.S "A visible light communication system for indoor application".International Journal of Engineering and Innovative Technology (IJEIT) Volume 3, Issue 12, June 2014

[2] Rajan Sagotra, Reena Aggarwal -"Visible light communication"International Journal of Engineering Trends and Technology Volume4Issue3-2013.

[3] Liju Sajan, Lince Mathew, Abraham Thomas, Sarun Sathyan, Bibin Baby "Wireless data transfer using visible light communication"

[4] Sumit Jaykant Meshram, Prof. Avinash P. Wadhe "Secure data transfer using visible light communication technique"International Journal of Innovative and Emerging Research in Engineering Volume 3, Special Issue 1, ICSTSD 2016.

[5] M.Sowmiya, G.Visali "A study on lifi wireless visible light communication" 2016 IJEDR | Volume 4, Issue 1 | ISSN: 23219939.

[6] Amit Agrawal, Gaurav kumar, Maheshwari narayan singh, Pradeep kumar, Pransu mathur "Data transmission using laser light" International Journal of Advanced Computer Technology (IJACT) ISSN:2319-7900.

[7] Rahul R. Sharma, Raunak, Akshay Sanganal "Li-fi technology transmission of data through light" Int.J.Computer Technology \& Applications, Vol 5 (1),150-154.

[8] R. Karthika, S. Balakrishnan "Wireless communication using Li-fi technology" SSRG International Journal of Electronics and Communication Engineering (SSRG-IJECE) volume 2 Issue 3 March 2015.

[9] P. A. Haigh, F. Bausi, T. Kanesan, T. Le, S. Rajbhandari, Z. Ghassemlooy, I. Papakonstantinou, W. O. Popoola, A. Burton, H. Le Minh, A. D. Ellis and F. Cacialli "A $10 \mathrm{mb} / \mathrm{s}$ visible light communication system using a low bandwidth polymer lightemitting diode" 2014 9th International Symposium on Communication Systems, Networks \& Digital Sign (CSNDSP). 